\title{
Improving the Nutritional Functionalities of Unimix Porridge by Lactic Fermentation Using Uji Starter Culture
}

\author{
Samuel Kuria Mbugua ${ }^{* 1}$, Jacinta Muchiri ${ }^{1}$ and Barugahara Evyline Isingoma ${ }^{1,2}$ \\ ${ }^{1}$ Department of Food Science, Nutrition and Technology, P.O. Box 29053 - 00625, University of Nairobi, Kenya \\ ${ }^{2}$ Department of Human Nutrition and Home Economics, Box 1, Kyambogo University Uganda
}

\begin{abstract}
Received: 9 November, 2019; Accepted: 3 December, 2019; Published: 6 December, 2019
*Corresponding author: Barugahara Evyline Isingoma, Department of Human Nutrition and Home Economics, Box 1, Kyambogo University Uganda, Tel: +256782673 038, E-mail: bisingoma@gmail.com
\end{abstract}

\begin{abstract}
In 1992, UNImix porridge caused an outbreak of anorexia, vomiting and diarrhoea among children in North Eastern Kenya. This was attributed to Trypsin Inhibitors (Ti) and Haemagglutinins (Hgg) in kidney beans and was solved by replacing Kidney beans with soya beans and extrusion cooking. However, the improved UNImix porridge has been associated with cases of flatulence, diarrhoea and poor palatability among children of displaced refugees in Sudan, due to alpha-oligosaccharides in soybeans, which cannot be eliminated by extrusion-cooking but by lactic fermentation, which is also known to eliminate Ti and Hgg and improve other nutritional functionalities in porridges. This study was therefore aimed at replacing extrusion-cooking in UNImix production with lactic fermentation and determination of its effect on Ti and Hgg. A $3 \times 5$ factorial experiment was carried out to test effect of lactic fermentation and cooking on UNImix containing soybeans and kidney beans, and compare results with the extrudedcooked UNImix. All unfermented UNImix samples containing kidney beans tested positive for Ti and Hgg even after boiling for 60 minutes; including those processed by fine milling and drum drying in combination with boiling, but negative on autoclaving at $121^{\circ} \mathrm{C}$ for 15 min, extrusion-cooking at $100-140^{\circ} \mathrm{C}$ and roasting. Lactic Fermentation alone of UNImix with either kidney beans or soybeans failed to inactivate either Ti or Hgg. However, lactic fermentation combined with boiling for $3-5$ minutes eliminated Ti in UNImix samples containing soybeans but not Kidney beans, and tested positive for Hgg. It is concluded that Ti and Hgg from Kidney beans are more difficult to inactivate than those in Soya beans and were responsible for the diseases' outbreak in North Eastern of Kenya in 1992. That lactic fermentation in combination with cooking for 3-5 minutes before consumption can replace extrusion-cooking in UNImix containing soybeans on the basis of inactivation of Ti with benefit of eliminating alpha-oligosaccharides the causative agents for flatulence and diarrhoea, and improvement of palatability among other nutritional functionalities.
\end{abstract}

Key Words: UNImix porridge, Trypsin inhibitors, Haemagglutinins, LAB Uji culture and Lactic fermentation.

\section{Introduction}

UNImix porridge is the pioneer for the nutritional therapeutic porridges. It was developed as an emergency ration for UNICEF Somalia in 1992 to cater for nutrition of displaced children and women in North Eastern Kenya, following overthrow of Somalia government in a military coup in 1991. The initial UNImix porridge consisted of a composite flour of maize and kidney beans fortified with vegetable oil and sugar. It was developed by the Nutrition Department of Ministry of Health of Kenya government. Consumption of this porridge by children in the North Eastern Kenya caused a massive outbreak of anorexia, vomiting and diarrhea. This led to UNICEF Somalia country office based at Gigiri, Nairobi Kenya at the time, to sanction investigations on causes of the outbreak and to provide solutions through improvement and development by the Department of Food Science, Nutrition and Technology, University of Nairobi [1].

Results from investigations pointed to inadequate inactivation/elimination of antitrypsin and hemagglutinin factors in the UNImix porridge containing kidney beans, and thus established grounds for the development of the present day UNImix porridge which contains soybeans instead of kidney beans and is extruded-cooked to inactivate trypsin inhibitors and haemagglutinins in the soybeans [1].

Since then several therapeutic porridge rations and complementary foods have been developed along the porridge concept for nutritional intervention and rehabilitation such as WFP mix by the World food program and Corn Soy Blend (CSB) from USA by USAID. The use of porridge rations for nutritional intervention and rehabilitation programs across Sub-Saharan region of Africa in refugee camps, famine distress spots and with people living with HIV virus has been a great success in countries such as Somalia, Kenya, Uganda, Democratic Republic of Congo (DRC), Ethiopia, South Sudan, Darfur region of Sudan, Malawi, Mozambique and Angola [2].

However, Nutritionists in Darfur region of Sudan reported cases of flatulence and diarrhea, including reduced UNImix porridge intake by children due to its low palatability. Flatulence and diarrhea can occur in some individuals caused by alphaoligosaccharides namely raffinose, verbascose and stachyose found in legumes like soybeans. Unlike Trypsin inhibitors (Ti) and haemagglutinins (Hgg) also found in legumes, alphaolygosaccharides cannot be eliminated by extrusion-cooking in 
production of UNImix porridge [3]. The alpha-oligosaccharides can however be eliminated by lactic fermentation through hydrolysis using appropriate Lactic Acid Bacteria (LAB) strains such as in Uji starter culture reported to also inactivate trypsin inhibitors and hemagglutinins [4]. The Uji culture exhibited invitro antimicrobial properties against some diarrheal causing pathogens but not insitu [8]. Several strains of Lactobacillus plantarum strains and especially those studied in some fermented milk products and porridges have been shown to have some probiotic properties.

Lactic fermentation can simultaneously impart several other beneficial nutritional functionalities in UNImix porridge such as improved palatability due to developed appealing taste and flavors, cook ability, protein and starch digestibility, minerals' bioavailability, lowered dietary bulk, and antimicrobial properties [5-10]. Minerals' bioavailability is made possible by LAB strains with phytase activity, while improved palatability increases porridge intake critical for enhancing convalescence among clinically malnourished children [3, 10 -12].

This study was therefore aimed at substituting extrusioncooking with lactic fermentation in UNImix porridge production, with special regard to inactivation/elimination of trypsin inhibitors and hemagglutinins using Uji (LAB) starter culture dominated with strains of Lactobacillus plantarum, some strains of Pediococcus acidilactici and Pediococcus pentosaceus known to eliminate by hydrolysis alpha-oligosaccharides and improve palatability of porridges $[4,5]$

\section{Methods}

\section{Sourcing of Raw Materials}

Maize, Soya bean and Kidney bean flours were obtained from Proctor and Allan Co. Ltd Nairobi, while Soybeans and flakes were obtained from Soy Afric.Co. Ltd Nairobi, having been imported from Ohio, U.S.A. The two companies were contracted by UNICEF Somalia to manufacture UNImix porridge ration.

\section{Fermentation of Unimix}

Slurry samples of UNImix porridge were fermented using Uji starter culture prepared according to Mbugua 1992 [13]. Optimal conditions used for fermentation were ambient temperatures ranging from about $22^{\circ} \mathrm{C}-35^{\circ} \mathrm{C}$ in keeping with traditional fermentation. Uji culture a mesophilic starter culture was developed, from the traditional fermentation process, and reduced traditional fermentation time from about 72 to $24 \mathrm{hrs}$.

Fermented slurry samples were prepared by mixing flour blends of UNImix with tap water at $40 \% \mathrm{w} / \mathrm{w}$ flour solids, inoculating with $U j i$ starter culture and incubating at $25 \mathrm{oC}$ for $24 \mathrm{hrs}$ in a $3 \times$ 5 factorial design, according to Figure 1. Fermented samples: K1 - K5, S1 - S5 and Sf1 - Sf5, were analysed for presence of Trypsin Inhibitors (Ti) and Haemagglutinins (Hgg) without boiling and after boiling for 3, 5, 10, and 15 minutes.

\section{Boiling of Samples}

The fermented UNImix samples with $40 \%$ flour solids were diluted five times to $8 \%$ solids in $125 \mathrm{ml}$ volumes in aluminum cups measuring 6.2 and $7.0 \mathrm{~cm}$ of diameter and height respectively and with lids. This helped achievement of appropriate consistency and viscosity for consumption. The slurry samples were brought to boil on a hot plate while stirring, covered and allowed to simmer for $3,5,10$, and 15 minutes and cooled to $25 \mathrm{oC}$ before analysis for Ti and Hgg. The boiling temperature for the slurries was 930C, the boiling and cooking temperature in Nairobi due to the attitude, but higher or lower temperatures due attitudes would not matter as long as temperatures do not drop below starch gelatinisation of $720 \mathrm{C}$ only possible in inhabitable high mountains.

\section{Detection of Trypsin Inhibitors (Ti)}

Trypsin inhibitors were detected by a reaction between crude protein extract from slurry samples and gelatin in the presence of trypsin enzyme according to Reddy et al 1986 [3]. The crude protein was extracted from samples by mixing $5 \mathrm{~g}$ of slurry with $100 \mathrm{ml}$ of $0.1 \mathrm{~N}$ sodium acetate, shaken for $2 \mathrm{hr}$. in a shaker incubator at $30^{\circ} \mathrm{C}$, centrifuged and the supernatant used as testing sample. $2 \mathrm{mg}$ trypsin enzyme powder was dissolved in $50 \mathrm{ml}$ of sodium phosphate buffer at $\mathrm{pH}$ 7.5. The reaction mixture involved $250 \mathrm{mg}$ gelatin, $5 \mathrm{ml}$ trypsin solution and 5 $\mathrm{ml}$ crude protein extract solution mixed to dissolve in a vortex mixer, incubated at $37^{\circ} \mathrm{C}$ for $2 \mathrm{~h}$. and then cooled to $4^{\circ} \mathrm{C}$. Samples containing trypsin inhibitors solidified in 15 minutes, while those without remained liquid indefinitely due to hydrolysis of gelatin by the added trypsin enzyme which was not inhibited. Control sample results for protein extracts from raw flour were used to confirm the results.

\section{Detection of Haemagglutinins (Hgg)}

Haemagglutinins were again detected according to Reddy et, al 1986 in the crude protein extracts [3]. The crude protein extract solution in $0.1 \mathrm{~N}$ sodium acetate was precipitated by mixing in $1: 1$ ratio with $6 \mathrm{M}$ sodium hydrogen sulphate solution, followed by separation by centrifugation. The precipitate was re-dissolved in $0.1 \mathrm{~N}$ sodium acetate. $10 \mathrm{ml}$ of this solution were mixed with $1 \mathrm{ml}$ of blood from rabbit in saline solution. Precipitation and haemolysis of the red blood cells were checked as evidence of presence of haemagglutinins. Control sample results for protein extracts from raw bean flour were used to confirm the results.

\section{Results}

Trypsin Inhibitors (Ti) and Haemagglutinins (Hgg) in Cooked Unimix Porridge Containing Kidney Bean and Pigeon Pea Flours

Table 1 shows the effect of cooking unfermented raw UNImix containing kidney beans, kidney beans protein extracts and pigeon peas on Trypsin Inhibitors (Ti) and Haemagglutinins (Hgg). Presence of both trypsin inhibitors (Ti) and haemagglutinins (Hgg) persisted even after boiling for 60 minutes, but were undetectable after autoclaving at $121^{\circ} \mathrm{C}$ for 15 minutes. 


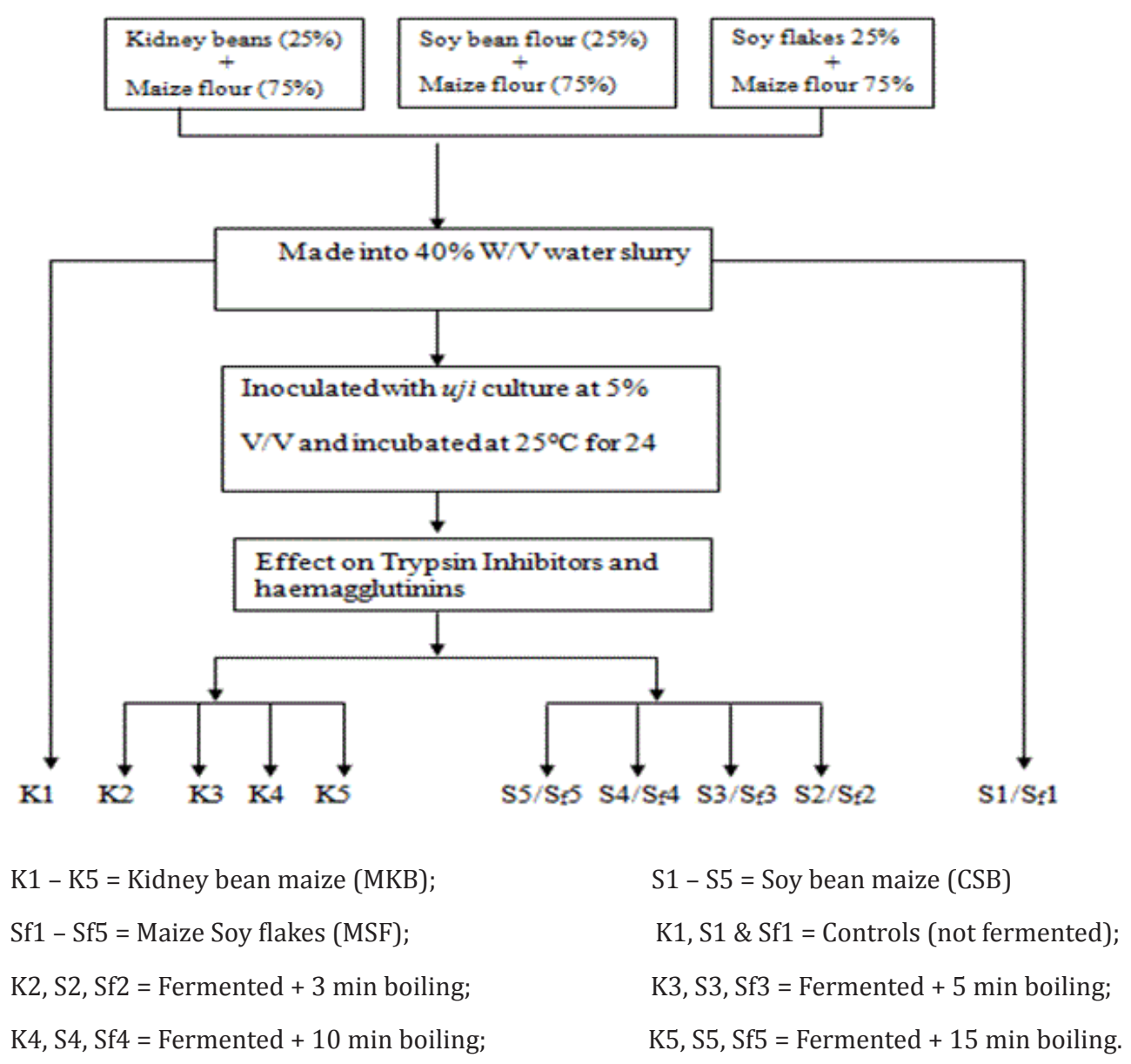

Figure 1: Research design

Table 1: Heat stability of Trypsin inhibitors (Ti) and Haemagglutinins (Hgg) in UNImix containing Kidney beans

\begin{tabular}{|l|c|c|c|c|}
\hline \multirow{2}{*}{ Bean Samples } & \multicolumn{2}{|c|}{60 min boiling at $93^{\circ} \mathrm{C}$} & \multicolumn{2}{|c|}{15 min autoclaving at $121^{\circ} \mathrm{C}$} \\
\cline { 2 - 5 } & Ti & Hgg & Ti & - \\
\hline All bean flours & + & + & - & - \\
\hline Bean protein extract & + & + & - & - \\
\hline Pigeon peas & + & + & - & \\
\hline \multicolumn{2}{|c|}{ Ti = Trypsin inhibitor; Hgg = Haemagglutinins; + Presence; - Absence } \\
\hline
\end{tabular}

\section{Effect of Selected Processing Treatments of Unimix Containing Kidney Beans on Ti and Hgg}

Table 2 shows the effect of roasting, drum drying, fine milling and boiling for 15 minutes and extrusion cooking at various temperatures on Ti and Hgg. Roasting inactivated Ti and Hgg completely, but created undesirable off-flavour and discoloration. Extrusion cooking inactivated Ti and Hgg at temperatures above 1000C, but left traces of Hgg which were eliminated only at 1400C. Both drum drying and fine milling together with boiling for 15 min were ineffective in elimination of the two nutritional stress factors. 
Table 2: Effect of various processing treatments on UNImix with kidney beans combined with boiling for 15 minutes on Ti and Hgg

\begin{tabular}{|c|c|c|c|c|}
\hline \multicolumn{5}{|l|}{ Processing } \\
\hline Method & $\mathrm{Ti}$ & Hgg & Off- flavour & Discolouration \\
\hline Roasting & - & - & +++ & +++ \\
\hline Extrusion cooking $\left(80^{\circ} \mathrm{c}\right)$ & + & + & - & - \\
\hline Extrusion cooking $\left(100^{\circ} \mathrm{C}\right)$ & - & \pm & - & - \\
\hline Extrusion cooking $\left(140^{\circ} \mathrm{c}\right)$ & - & - & - & - \\
\hline Drum drying & + & + & - & - \\
\hline Fine milling & + & + & - & - \\
\hline
\end{tabular}

Ti = Trypsin inhibitor; Hgg = Haemagglutinins; - = Absent; + = Present; + = slightly present; + + + = Intensive development

Source: Mbugua S and J Njenga. The antimicrobial activity of fermented uji. Ecology of food and nutrition. 1992;28(3):191-198.

\section{Effect of Lactic Fermentation of Unimix Porridge on Trypsin Inhibitors and Haemagglutinins}

The effect of lactic fermentation of UNImix porridge on Trypsin Inhibitors (Ti) and haemagglutinins (Hgg) is shown in Table 3. The UNImix samples had their pH reduced to 3.0-3.2 and titratable acidities increased to between $0.8-1.0 \%$ lactic acid equivalent on fermentation. There were no significant differences in these parameters between the samples. Fermentation alone without boiling did not affect either Ti or Hgg, while Hgg persisted in all fermented samples irrespective of cooking time. Trypsin inhibitors in UNImix containing soybeans and soybeans flakes were eliminated by a combination of fermentation and boiling for 3 and 5 minutes respectively. However, presence of trypsin inhibitors in fermented UNImix containing kidney beans persisted even after boiling for up to 15 minutes.

\begin{tabular}{|c|c|c|c|c|c|c|}
\hline \multirow[t]{2}{*}{$\begin{array}{l}\text { Cooking } \\
\text { Time }\end{array}$} & \multicolumn{2}{|c|}{$\begin{array}{l}\text { Soy flakes maize } \\
\text { (MSF) }\end{array}$} & \multicolumn{2}{|c|}{$\begin{array}{c}\text { Soy bean maize } \\
(\mathrm{CSB})\end{array}$} & \multicolumn{2}{|c|}{$\begin{array}{l}\text { Kidney bean maize } \\
\text { (MKB) }\end{array}$} \\
\hline & $\mathrm{Ti}$ & Hgg & $\mathrm{Ti}$ & Hgg & $\mathrm{Ti}$ & $\mathrm{Hgg}$ \\
\hline 3 & + & + & - & + & + & + \\
\hline 5 & + & + & - & + & + & + \\
\hline 10 & - & + & - & + & + & + \\
\hline 15 & - & + & - & + & + & + \\
\hline $\mathrm{pH}$ & \multicolumn{2}{|c|}{3.20} & \multicolumn{2}{|c|}{3.10} & \multicolumn{2}{|c|}{3.00} \\
\hline $\mathrm{TA}$ & \multicolumn{2}{|c|}{0.80} & \multicolumn{2}{|c|}{0.90} & \multicolumn{2}{|c|}{1.00} \\
\hline
\end{tabular}

\section{Discussion}

The study established that Ti and Hgg in kidney beans were either extremely heat stable or in such quantities unable to be completely inactivated by boiling for up to 60 minutes, but by autoclaving for 15 minutes at $121^{\circ} \mathrm{C}$; hence the conclusion that the Ti and Hgg in the UNImix containing Kidney beans and cooked for 15 minutes were responsible for the outbreak of anorexia, vomiting and diarrhoea among the children fed on the porridge in North Eastern Kenya. The ensuing developmental studies with treatments aimed at inactivating Ti and Hgg namely; drum drying and fine milling combined with boiling for 15 minutes were ineffective in eliminating $\mathrm{Ti}$ and Hgg, except for roasting and extrusion cooking. Roasting unfortunately caused discolouration and off-flavours in the UNImix porridge due to maillard reaction known to lower protein digestibility in porridges [14]. Extrusioncooking on the other hand at temperature of $140^{\circ} \mathrm{C}$ eliminated both $\mathrm{Ti}$ and Hgg without creation of discolouration or offflavours. Accordingly, all factories contracted to manufacture UNImix porridge by UNICEF Somalia namely; Proctor and Allan Ltd Nairobi, Soy Afric Ltd Nairobi and House of Manji Co Ltd Nairobi were required to use soybeans instead of kidney beans for protein fortification, and pre-cook it by extrusion at $140 \mathrm{oC}$ to ensure safety from Ti and Hgg [1].

The presence of Hgg in UNImix porridge persisted irrespective of processing treatments with exception of extrusion-cooking and roasting, whether it contained kidney beans or soybeans. In 
particular, boiling of UNImix porridge containing kidney beans for up to 2 hours failed to eradicate its presence an indication of unlikelihood of its elimination in recipes containing kidney beans and cooked under normal households' procedures [1]. This raises questions on harmfulness of Hgg on ingestion, given that no problems have been reported in communities consuming such diets. Reddy et al 1986 reported no problems associated with consumption of kidney beans diets cooked and processed differently but with detectable Hgg [3]. This fact can indirectly be collaborated by reported improvement of rehabilitative value and efficacy of High Energy porridge (HEP) containing kidney beans by lactic fermentation and presumably with residues of Hgg after cooking on clinically malnourished children in Uganda [11].

Current studies on replacement of extrusion-cooking with lactic fermentation using Uji starter culture showed that fermentation alone and even in combination with boiling of UNImix porridge containing kidney beans for up to 15 minutes was unable to eliminate Ti and Hgg, again demonstrating either stability of Ti and Hgg in kidney beans against inactivation under these conditions or their presence in such high quantities in Kidney beans requiring more time for inactivation at the given temperature. However, the Ti in the lactic fermented UNImix porridge containing soybeans but not Hgg was eliminated within 3-5 minutes, implying either more heat labile Ti in Soybeans than those in Kidney beans or their presence in such lower levels requiring shorter time for inactivation on basis of their inactivation kinetics. This finding however, justifies replacement of extrusion-cooking with lactic fermentation in production of UNI mix containing soybeans on the basis of elimination of Ti on minimal boiling for 3-5 minutes, considering that any residue Hgg are apparently harmless. Coincidentally lactic fermentation is reported to improve cook ability of porridges by enhancing starch gelatinization cycles during boiling thus lowering energy requirement unlike in extrusion-cooking, and making it appropriate for production of UNImix porridge at house hold level for nutritional intervention in children by mothers in Africa [7, 12-14].The fact that strains of Leuconostoc mesenteroides involved in lactic fermentation of Idli (an Indian legume-based product) have been reported to inactivate Hgg is a clear indication of diversity of LAB strains which can be harnessed and formulated in to desirable starter cultures for production of therapeutic porridge rations like UNImix porridge with desirable nutritional functionalities, which can be produced at household level in Africa for nutritional intervention and rehabilitation on sustainable basis instead of relying on industrially extruded-cooked porridge rations through donations [3, 10, 15-17].

\section{Conclusion}

This study has proved that it is possible to replace the extrusioncooking process with lactic fermentation using appropriate LAB starter cultures in production of UNImix porridge and related therapeutic porridge rations with profound benefits of improvement of nutritional functionalities of the porridge rations, several of which cannot be provided by extrusion- cooking. Additionally, lactic fermentation would make it feasible to produce therapeutic porridge rations on house hold basis and thus empower mothers in Africa technologically to produce such porridge rations using locally available food substrates for nutritional intervention and rehabilitation of their children on a sustainable basis, without relying on donated industrially produced porridge rations.

\section{Acknowledgement}

We wish to acknowledge UNICEF Somalia country office, Nairobi who in 1992 provided funds for investigations and development of UNImix;Department of |Food Science, Nutrition and Technology, University of Nairobi for availing analytical laboratory facilities and Proctor and Allan Company Ltd for use of their Extrusion machinery.

\section{References}

1. Mbugua SK, Keya EL. UNImix quality evaluation and development. A report for UNICEF Somalia Country Office, Nairobi. Department of Food Technology and Nutrition, University of Nairobi.

2. Maritim G, Mason J. 1994. Report of a workshop on the improvement of the nutrition of refugees and displaced people in Africa. Machakos, Kenya, 5 - 7 December 1994. An initiative by UNACC Sub- Committee on Nutrition, UNHCR, WFP, Applied Human Nutrition (ANP) OF University of Nairobi and GTZ- Germany.

3. Reddy NR, Pierson MD, Salunkhe DK. Legume based Fermented Foods. CRC Press Inc. U.S.A. 1986;155-170.

4. Mbugua SK, Ledford RA and Steinkraus KH. Gaschromatographic determination of mono-, di- and trisaccharides in uji flour ingredients during fermentation. Chem-Microbiol Technol. Lebensm. 1983;8:4045.

5. Onyango C, Okoth MW, Mbugua SK. Effect of drying lactic fermented uji (an East African sour porridge) on some carboxylic acids. J Sci Food Agric. 2000;80(13):1854-1858.

6. Barugahara Evyline Isingoma, Mbugua K Samuel, Karuri Edward, and Gakenia Wamuyu Maina. Socioeconomic and Demographic Factors Influencing Feeding Practices, Morbidity Status, and Dietary Intakes of Children Aged 7-24 Months in Rural Uganda. Ecology of Food and Nutrition. 2017;56(1):1-16.

7. Mbugua SK. Nutritional and fermentation aspects of "Uji" produced from dry milled maize flour (Unga baridi) and wet milled whole maize. Chem. Mikrobiol. Technol. Lebensm. 10:154-161.

8. Mbugua SK and Njenga J. The antimicrobial activity of fermented Uji. Ecology of Food and Nutrition. 1992;28(3): 191-198.

9. Batista NN, Ramos CL, de Figueiredo Vilela L, Dias DR, Schwan RF. Fermentation of yam (Dioscorea spp. L.) by indigenous phytase-producing lactic acid bacteria strains. Braz J Microbiol. 2019;50(2):507-514. doi: 10.1007/s42770-019-00059-5

10. Lorri WSM. Nutritional and microbiological evaluation of fermented cereal weaning foods. Ph.D. Thesis Dept of Food Science. Chalmers University of Technology Goteborg Sweden. 1993.

11. Namayengo FM 2001. Substitution of High Energy Milk (HEM) with High Energy-High Protein Lactic Fermented kidney bean -maize 
porridge (HEP), in rehabilitation of clinically malnourished children at Mlango Hospital, Uganda. MSc Thesis Dept. Food Technology and Nutrition. University of Nairobi.

12. Barugahara Evyline Isingoma, Mbugua K Samuel, Karuri G Edward and Gakenia W Maina. Performance of Nutritionally Optimized Millet Porridges in the Rehabilitation of Severely Malnourished Children at Mulango National Referral Hospital, Uganda. Journal of Advances in Medicine \& Medical Research. 2016;18(2): 1-12.

13. Mbugua SK. A method for manufacture of a fermented cereal product, African Research and Invention Property Office (ARIPO) Patent No. AP 122, 30.4.1991 and U.K Patent G.B 2.225 922B, 20.6.1992

14. Mbugua SK, Ahrens RA, Kigutha HN, Subramanian V. Effect of Fermentation, malt flour treatment and drum drying on nutritional quality of Uji. Ecology of Food and Nutrition. 1992;28(4):271-277.

15. Yao AA, Dortu C, Egounlety M, Pinto C, Edward VA, Huch M, et al. Production of freeze-dried Lactic Acid Bacteria starter cultures for cassava fermentation in to Gari. African Journal of Biotechnology. 2009;8(19):4996-5004.

16. Holpzafel W. Use of starter cultures in fermentation on a household scale. Food Control. 1997:8(5-6):241-258.

17.WHO,1996:Fermentation- Assessment and Research; Report of a joint FAO/WHO Workshop on fermentation as a household technology to improve safety, in collaboration with the Department of Health, Republic of South Africa Pretoria, South Africa, 11 - 15 December 1995. Food Safety Unit, Division of Food and Nutrition, WHO. 1996. 